\title{
SUBORDINATING FACTOR SEQUENCES FOR CONVEX MAPS OF THE UNIT CIRCLE
}

\author{
HERBERT S. WILF ${ }^{1}$
}

I. Introduction. Let $K$ denote the class of functions

$$
f(z)=\sum_{\nu=1}^{\infty} a_{\nu} z^{\nu}
$$

which are regular in the unit circle and map it onto a schlicht convex domain. In a recent paper [1] Pólya and Schoenberg have shown that in order for $f(z) \in K$ it is necessary and sufficient that each of the functions

$$
V_{n}(z ; f)=\frac{1}{C_{2 n, n}} \sum_{\nu=1}^{n} C_{2 n, n+\nu} a_{v} z^{\nu} \quad(n=1,2, \cdots)
$$

belong to $K$.

We will use the notation

$$
f(z) \subseteq g(z)
$$

(" $f(z)$ is subordinate to $g(z)$ ") to mean that $f(z), g(z)$ are both regular in $|z|<1$, that $g(z)$ is univalent there, and that every value taken by $f(z)$ in $|z|<1$ is also taken by $g(z)$ (see $[2 ; 3]$ ). It was shown in [1] that

$$
V_{n}(z ; f) \subseteq f(z)
$$$$
(n=1,2, \cdots)
$$

for every $f(z) \in K$, and it was pointed out that even

$$
V_{1}(z ; f) \subseteq V_{2}(z ; f) \subseteq \cdots \subseteq f(z)
$$

is likely, though this was not verified except for

$$
f_{0}(z)=z(1-z)^{-1} \text {. }
$$

In the following paragraphs we will show how consideration of the problem (5) leads, in a natural way, to the question of characterizing certain kinds of factor sequences (see [6]), and although we cannot decide the truth or falsity of (5), a closely related question will be completely settled (Theorem 2 , infra).

Received by the editors July 22, 1960 and, in revised form, September 9, 1960.

1 This work supported by the National Science Foundation. 
II. Subordinating factor sequences. An infinite sequence $\left\{b_{v}\right\}_{1}^{\infty}$ of complex numbers will be called a subordinating factor sequence if whenever

$$
f(z)=\sum_{v=1}^{\infty} a_{v} z^{y} \in K
$$

we have

$$
\sum_{v=1}^{\infty} a_{v} b_{v} z^{\nu} \subseteq f(z)
$$

A finite sequence $\left\{b_{\nu}\right\}_{1}^{n}$ will be called a subordinating factor sequence if (7) implies (8) whenever $a_{n+1}=a_{n+2}=\cdots=0$. The class of such infinite sequences we denote by $F$, and that of sequences of length $n$ by $\mathfrak{F}_{n}$.

THEOREM 1. The proposition

$$
\left\{1-\frac{\nu^{2}}{n^{2}}\right\}_{\nu=1}^{n} \in F_{n}
$$

implies (5).

PROOF. This is immediate from the easily established identity

(10) $\left(1+\frac{z}{n} \frac{d}{d z}\right)\left(1-\frac{z}{n} \frac{d}{d z}\right) V_{n}(z ; f)=V_{n-1}(z ; f) \quad(n=2,3, \cdots)$

and the definition of $\mathcal{F}_{n}$.

We do not know how to characterize sequences of $\mathscr{F}_{n}$. The following result, however, completely describes the class $\mathcal{F}$.

THEOREM 2. The following three properties of a sequence of complex numbers are equivalent:

(I) $\quad\left\{b_{v}\right\}_{1}^{\infty} \in \mathcal{F}$;

(II) $\operatorname{Re}\left\{1+2 \sum_{\nu=1}^{\infty} b_{\nu} z^{\nu}\right\}>0$ $(|z|<1)$

(III) $b_{\nu}=\frac{1}{2 \pi} \int_{0}^{2 \pi} e^{i \nu \theta} d \psi(\theta)$ $\left(\nu=0,1,2, \cdots ; b_{0}=1 ; \psi(\theta) \uparrow\right)$.

Proof. The equivalence of (II) and (III) is classical. Now suppose (I) holds. Then 


$$
\sum_{\nu=1}^{\infty} b_{\nu} z^{\nu} \subseteq \sum_{\nu=1}^{\infty} z^{\nu}=z(1-z)^{-1}
$$

which is to say that,

$$
\operatorname{Re}\left\{\sum_{\nu=1}^{\infty} b_{\nu} z^{\nu}\right\}>-\frac{1}{2} \quad(|z|<1),
$$

which proves (II). Conversely, if (III) holds, let

$$
f(z)=\sum_{\nu=1}^{\infty} a_{\nu} z^{\nu} \in K
$$

Then

$$
\begin{aligned}
\sum_{\nu=1}^{\infty} a_{\nu} b_{\nu} z^{\nu} & =\frac{1}{2 \pi} \int_{0}^{2 \pi} \sum_{\nu=1}^{\infty} a_{\nu} e^{i \nu \theta} r^{\nu} e^{i \nu \phi} d \psi(\theta) \\
& =\frac{1}{2 \pi} \int_{0}^{2 \pi} f\left(r e^{i(\theta+\phi)}\right) d \psi(\theta) .
\end{aligned}
$$

The left hand side is thus exhibited as the centroid of a nonnegative mass distribution of total mass one, on a convex curve, and therefore lies inside that curve, which was to be shown.

Several results, some well known, follow immediately from Theorem 2 .

Corollary 1. If $\left\{b_{\nu}\right\}_{1}^{\infty} \in \mathcal{F},\left\{c_{\nu}\right\}_{1}^{\infty} \in \mathcal{F}$, then $\left\{b_{\nu} c_{\nu}\right\}_{1}^{\infty} \in \mathcal{F}$.

Since from (12), the result of applying a sequence of $\mathcal{F}$ to an arbitrary analytic function is a function which maps the unit circle into the convex hull of the original image, the result of applying these two sequences to $f(z)$ in succession is clearly subordinate to $f(z)$.

Corollary 2. If

$$
\operatorname{Re}\left\{1+2 \sum_{1}^{\infty} a_{\nu} z^{\nu}\right\}>0, \quad \operatorname{Re}\left\{1+2 \sum_{1}^{\infty} b_{\nu} z^{\nu}\right\}>0
$$

then

$$
\operatorname{Re}\left\{1+2 \sum_{1}^{\infty} a_{\nu} b_{\nu} z^{\nu}\right\}>0 \quad(|z|<1) .
$$

This well-known result $[5, \mathrm{VII}, 43]$ is clear from Theorem 2 and Corollary 1.

CoRollary 3. The image of the unit circle under the mapping 


$$
f(z)=z+\sum_{\nu=2}^{\infty} a_{\nu} z^{\nu}
$$

of $K$, contains the circle $|W|<1 / 2$, the constant being sharp.

This result, due to Study [4] (compare [2, p. 223]; [1, p. 320]) is precisely the assertion that the sequence $1 / 2,0,0, \cdots$ belongs to $\mathcal{F}$, which is obvious from Theorem 2, (II). The sharpness is shown, as usual, by the example (6).

Corollary 4. Equation (4) is true.

Indeed, from (6) and $\left(6^{\prime}\right)$ of [1] with $z=e^{i \theta}$, there follows

$$
\begin{aligned}
\operatorname{Re}\left\{1+2 V_{n}\left(z ; \frac{z}{1-z}\right)\right\} & =1+2 \sum_{\nu=1}^{n} \frac{n !}{(n-\nu) !} \frac{n !}{(n+\nu) !} \cos \nu \theta \\
& =\frac{(n !)^{2}}{(2 n) !}\left(2 \cos \frac{\theta}{2}\right)^{2 n} \\
& \geqq 0
\end{aligned}
$$

whence the sequence

$$
b_{\nu}=\left\{\begin{array}{cr}
C_{2 n, n+\nu} / C_{2 n, n} & (\nu=1,2, \cdots, n) \\
0 & (\nu \geqq n+1)
\end{array}\right.
$$

belongs to $F$, which is exactly what (4) asserts (our proof is really identical with that in [1]).

Corollary 5. Let the functions $f(z)=\sum_{1}^{\infty} a_{\nu} z^{\nu}, g(z)=\sum_{1}^{\infty} b_{\nu} z^{\nu}$ belong to $K$, and map $|z|<1$ onto domains $\mathfrak{D}^{\prime}$, $\mathfrak{D}^{\prime \prime}$, respectively, both contained in $\operatorname{Re} w>-1 / 2$. Then the function $\sum_{1}^{\infty} a_{\nu} b_{\nu} z^{\nu}$ maps $|z|<1$ onto a domain $D \subseteq D^{\prime} \cap D^{\prime \prime}$.

This result, which is related to a conjecture of Pólya-Schoenberg on the Hadamard product of functions of $K$, follows by noting that $\left\{a_{\nu}\right\}_{1}^{\infty} \in \mathcal{F} \Rightarrow D \subseteq D^{\prime \prime}$, and $\left\{b_{\nu}\right\}_{1}^{\infty} \in \mathcal{F} \Rightarrow D \subseteq D^{\prime}$ which was to be shown.

Concerning the open question (9) we may now see that, in any event, the sequence $\left\{1-\nu^{2} / n^{2}\right\}_{1}^{n}$ is not extendable to a sequence of $\mathcal{F}$ since that would require the positivity of the Toeplitz matrix

$$
T=\left(\begin{array}{cccc}
1 & 1-1 / n^{2} & 1-4 / n^{2} & \ldots \\
1-1 / n^{2} & 1 & 1-1 / n^{2} & \ldots \\
1-4 / n^{2} & 1-1 / n^{2} & 1 & \ldots \\
\cdot & : & : &
\end{array}\right)
$$


whereas the $3 \times 3$ determinant in the upper left corner has the value $-8 n^{-6}<0$.

III. On the composition of convex maps. In [1] it was conjectured that if $f(z)=\sum a_{\nu} z^{\nu} \in K$ and $g(z)=\sum b_{\nu} z^{\nu} \in K$ then so does $h(z)$ $=\sum a_{v} b_{v} z^{\nu}$. We state here a single proposition whose truth would imply both this conjecture and (5) at once. It is

Proposition 1. The coefficients of a convex function preserve subordination between convex functions. That is, if $\sum a_{\nu} z^{\nu}, \sum b_{\nu} z^{\nu}, \sum c_{\nu} z^{\nu}$ are all in $K$, and if

$$
\sum a_{\nu} z^{\nu} \subseteq \sum b_{\nu} z^{\nu}
$$

then

$$
\sum a_{v} c_{\nu} z^{\nu} \subseteq \sum b_{v} c_{\nu} z^{\nu}
$$

Indeed, if this is true, then since (5) holds in the case (6) it holds in general. Further, by applying the sequence $\left\{b_{v}\right\}_{1}^{\infty}$ to the relations

$$
V_{n}(z ; f) \subseteq f(z) \quad(n=1,2, \cdots)
$$

we would find that the means of the function $\sum a_{v} b_{v} z^{v}$ are subordinate to the function itself, and in view of a recent result of Robertson [7], it would follow that $\sum a_{\nu} b_{\nu} z^{\nu} \in K$.

\section{REFERENCES}

1. G. Pólya and I. J. Schoenberg, Remarks on de la Vallée Poussin means and convex conformal maps of the circle, Pacific J. Math. vol. 8 (1958) pp. 295-334.

2. Z. Nehari, Conformal mappings, New York, McGraw-Hill, 1952.

3. J. E. Littlewood, Jr., Lectures on the theory of functions, Oxford University Press, 1944.

4. E. Study, Vorlesungen über ausgewählte Gegenstände der Geometrie, Second Part, Leipzig and Berlin, 1913.

5. G. Poblya and G. Szegö, Aufgaben und Lehrsätze aus der Analysis, Berlin, 1925.

6. G. Polya and I. Schur, Über zwei Arten von Faktorenfolgen in der Theorie der algebraischen Gleichungen, J. Reine Angew. Math. vol. 144 (1914) pp. 89-113.

7. M. S. Robertson, Applications of the subordination principle to univalent functions, Abstract 571-172, Notices Amer. Math. Soc. vol. 7 (1960) p. 641.

The University of ILlinois 